\title{
Neutrino mass ordering at DUNE: An extra $\nu$ bonus
}

\author{
Christoph A. Ternes $\odot,{ }^{*}$ Stefano Gariazzo, ${ }^{\dagger}$ Rasmi Hajjar, ${ }^{\ddagger}$ Olga Mena, ${ }^{\S}$ Michel Sorel,, and Mariam Tórtola \\ Instituto de Física Corpuscular CSIC-Universitat de València, 46980 Paterna, Spain
}

(Received 21 May 2019; published 21 November 2019)

\begin{abstract}
We study the possibility of extracting the neutrino mass ordering at the future Deep Underground Neutrino Experiment using atmospheric neutrinos, which will be available before the muon neutrino beam starts being operational. The large statistics of the atmospheric muon neutrino and antineutrino samples at the far detector, together with the baselines of thousands of kilometers that these atmospheric (anti) neutrinos travel, provide ideal ingredients to extract the neutrino mass ordering via matter effects in the neutrino propagation through Earth. Crucially, muon capture by argon provides excellent charge tagging, allowing us to disentangle the neutrino and antineutrino signature. This is an important extra benefit of having a liquid argon time projection chamber as a far detector, that could render an $\sim 3.5 \sigma$ extraction of the mass ordering after approximately $7 \mathrm{yr}$ of exposure.
\end{abstract}

DOI: 10.1103/PhysRevD.100.093004

\section{INTRODUCTION}

Neutrino oscillation experiments imply the first departure from the Standard Model of particle physics, as they have found overwhelming evidence for the existence of neutrino masses. Despite the accuracy they provide on the neutrino oscillation parameters-which is of the order of the percent level [1] - the sign of the atmospheric mass splitting, $\Delta m_{31}^{2}$, and the value of the $C P$-violating phase $\delta$ both remain unknown. The sign of $\Delta m_{31}^{2}$ originates two possible scenarios, normal (NO) or inverted ordering (IO) $[2,3]$. The sensitivity to the neutrino mass spectrum at oscillation experiments is mostly coming from the presence of matter effects [4-13] in the neutrino and antineutrino propagation. In the normal (inverted) mass ordering scenario, the neutrino flavor transition probabilities will get enhanced (suppressed), while in the case of antineutrino propagation the opposite happens and the antineutrino flavor transition probabilities will get suppressed (enhanced) in the normal (inverted) mass ordering scenario. At long-baseline accelerator experiments, matter effects, and, consequently, the sensitivity to the mass ordering, increase with the baseline, while these effects will be

\footnotetext{
*chternes@ific.uv.es

†gariazzo@ific.uv.es

rashajmu@alumni.uv.es

\$omena@ific.uv.es

"sorel@ific.uv.es

"mariam@ific.uv.es
}

Published by the American Physical Society under the terms of the Creative Commons Attribution 4.0 International license. Further distribution of this work must maintain attribution to the author(s) and the published article's title, journal citation, and DOI. Funded by SCOAP. negligible at short-baseline and medium-baseline experiments. Despite this, when extracting both the mass ordering and the $C P$-violating phase from results of long-baseline facilities, knowledge on the mixing angle $\theta_{13}$ in a vacuum is required. Short- and medium-baseline experiments at reactors have been fundamental to establish strong constraints on such an angle. Despite the fact that the neutrino mass ordering remains unknown, current oscillation data are mildly favoring the normal ordering scenario. The authors of Refs. [1,3] have reported a global preference for normal ordering at the level of $2.7 \sigma$ from all long-baseline accelerator and short-baseline reactor data (i.e., T2K, NO $\nu$ A, K2K, MINOS, Daya Bay, RENO, and Double Chooz).

The future long-baseline facility Deep Underground Neutrino Experiment (DUNE) [14-16] aims to extract the sign of the atmospheric mass splitting and the $C P$-violating phase $\delta$ through the golden channels $\nu_{\mu} \rightarrow \nu_{e}$ and $\bar{\nu}_{\mu} \rightarrow \bar{\nu}_{e}$, the same channels exploited by the current T2K $[17,18]$ and $\mathrm{NO} \nu \mathrm{A}[19,20]$ experiments. However, both quantities can also be extracted using atmospheric neutrino beams. ${ }^{1}$ Indeed, the idea of using atmospheric neutrino fluxes to distinguish the type of mass ordering has been well known in the literature for a long time $[23,24]$. These pioneer studies focused mostly on muon calorimeter detectors, such as MONOLITH [25], MINOS [26], or INO [27], in which the muon charge can be determined; see also Refs. [28-41]. Furthermore, in the absence of a chargedcurrent event by event final muon charge discrimination, the addition of the atmospheric neutrino oscillation data to the analysis performed in Ref. [1] improves the preference

\footnotetext{
${ }^{1}$ See Ref. [21] and the recent work of [22] for a $C P$-violation measurement using sub-GeV atmospheric neutrinos.
} 
for normal ordering to the level of $3.4 \sigma$, mostly due to the Super-Kamiokande $\nu_{\mu} \rightarrow \nu_{e}$ data sample [42], where the separation among $\nu_{e}$ and $\bar{\nu}_{e}$ events is done statistically.

Neutrino observatories can also extract the sign of the atmospheric mass difference with lower energy detection thresholds for atmospheric neutrino extensions by looking at the less sensitive but higher statistics muon disappearance channels such as $\nu_{\mu} \rightarrow \nu_{\mu}$ and $\bar{\nu}_{\mu} \rightarrow \bar{\nu}_{\mu}$ [43]. The IceCube Collaboration has recently reported a preference for NO with a $p$ value of $p_{\mathrm{IO}}=15.3 \%$ for the $\mathrm{IO}$ hypothesis [44] using data collected by the DeepCore extension. This will also be the main target for ORCA [45,46] and PINGU [47-50]; see, e.g., [51-58].

In this manuscript, we exploit the atmospheric neutrino signatures at the DUNE detector, a liquid argon time projection chamber (LArTPC). Despite this detection technology, in the absence of a magnetic field, not allowing for a charge identification of the final lepton state, one can make use of a particular event topology available in argon detectors: muon capture. This bonus process will provide a clean measurement of the muon charge that will considerably improve the capabilities of DUNE to perform mass ordering measurements with atmospheric neutrinos. Notice that the advantage is twofold, as (i) measurements of the mass ordering could be available before the beam starts, and (ii) the combination with the beam information will notably enhance the expected sensitivity reach. We shall show that muon capture events could greatly enhance the sensitivity to the mass ordering from atmospheric neutrinos only. For an earlier, and preliminary, appraisal of the neutrino mass ordering sensitivity in DUNE using atmospheric neutrinos, including a statistical discrimination between neutrinos and antineutrinos, see Ref. [59]. The latter work was largely based on previous studies in the framework of the LBNE project; see [60].

The structure of the paper is as follows: In Sec. II, we describe the oscillations of atmospheric neutrinos and the matter effects they undergo. Next, in Sec. III, we discuss the simulation of the neutrino event rates at the DUNE far detector and how the muon capture comes into play. Section IV contains the description of the statistical method and the main results obtained in this study. Our final remarks are presented in Sec. V.

\section{MATTER EFFECTS AND ATMOSPHERIC NEUTRINOS}

In atmospheric neutrino experiments, the size of matter effects is given by the effective mixing angle $\theta_{13}$ in matter, which leads to the golden channel transitions $\nu_{\mu} \rightarrow \nu_{e}$, $\nu_{e} \rightarrow \nu_{\mu}, \bar{\nu}_{\mu} \rightarrow \bar{\nu}_{e}$, and $\bar{\nu}_{e} \rightarrow \bar{\nu}_{\mu}$ and reads, within the simple two-flavor mixing framework, as

$$
\sin ^{2} 2 \theta_{13}^{\mathrm{m}}=\frac{\sin ^{2} 2 \theta_{13}}{\sin ^{2} 2 \theta_{13}+\left(\cos 2 \theta_{13} \mp \frac{A}{\Delta m_{31}^{2}}\right)^{2}},
$$

where the minus (plus) sign refers to neutrinos (antineutrinos). The matter potential is given by $A=2 \sqrt{2} G_{F} N_{e} E$, and $N_{e}$ is the electron number density in Earth's interior. Consequently, matter effects will enhance (deplete) the neutrino (antineutrino) oscillation probabilities $P\left(\nu_{\mu} \rightarrow \nu_{e}\right)$ and $P\left(\nu_{e} \rightarrow \nu_{\mu}\right)\left[P\left(\bar{\nu}_{\mu} \rightarrow \bar{\nu}_{e}\right)\right.$ and $\left.P\left(\bar{\nu}_{e} \rightarrow \bar{\nu}_{\mu}\right)\right]$ if the mass ordering is normal. When the resonance condition

$$
\Delta m_{31}^{2} \cos 2 \theta_{13}=2 \sqrt{2} G_{F} N_{e} E
$$

is satisfied, matter effects are expected to have their largest contribution. In the case of atmospheric neutrinos, which travel distances of several thousand of kilometers, and for $\Delta m_{31}^{2} \sim 2.5 \times 10^{-3} \mathrm{eV}^{2}$ [1], the resonance condition will take place at neutrino energies $\sim 3-8 \mathrm{GeV}$, depending on the precise value of $N_{e}$ in Earth's interior.

Matter effects are also present in the muon disappearance channels $P\left(\nu_{\mu} \rightarrow \nu_{\mu}\right)$ and $P\left(\bar{\nu}_{\mu} \rightarrow \bar{\nu}_{\mu}\right)$, relevant for both long-baseline and atmospheric neutrino beams. In the simplified case of a constant matter density, the disappearance probability at terrestrial baselines ${ }^{2}$ is given by

$$
\begin{aligned}
P\left(\stackrel{(-)}{\nu_{\mu}} \rightarrow \stackrel{(-)}{\nu_{\mu}}\right)= & 1-\cos ^{2} \theta_{13}^{\mathrm{m}} \sin ^{2} 2 \theta_{23} \\
& \times \sin ^{2}\left[1.27\left(\frac{\Delta m_{31}^{2}+A+\left(\Delta m_{31}^{2}\right)^{\mathrm{m}}}{2}\right) \frac{L}{E}\right] \\
& -\sin ^{2} \theta_{13}^{\mathrm{m}} \sin ^{2} 2 \theta_{23} \\
& \times \sin ^{2}\left[1.27\left(\frac{\Delta m_{31}^{2}+A-\left(\Delta m_{31}^{2}\right)^{\mathrm{m}}}{2}\right) \frac{L}{E}\right] \\
& -\sin ^{4} \theta_{23} \sin ^{2} 2 \theta_{13}^{\mathrm{m}} \sin ^{2}\left[1.27\left(\Delta m_{31}^{2}\right)^{\mathrm{m}} \frac{L}{E}\right],
\end{aligned}
$$

where $\theta_{13}^{\mathrm{m}}$ is that of Eq. (1) and

$$
\left(\Delta m_{31}^{2}\right)^{\mathrm{m}}=\Delta m_{31}^{2} \sqrt{\sin ^{2} 2 \theta_{13}+\left(\cos 2 \theta_{13} \mp \frac{A}{\Delta m_{31}^{2}}\right)^{2}} .
$$

The muon survival probabilities will be suppressed (enhanced) if the ordering is normal (inverted), so the effect is opposite to the one present in the $\nu_{e} \rightarrow \nu_{\mu}$ oscillation channel. Therefore, when dealing with atmospheric neutrino beams, since there is an irreducible muon neutrino background from $\nu_{e} \rightarrow \nu_{\mu}$ oscillations, the size of the matter effects will be reduced. The distance $L$ traveled through Earth by these atmospheric neutrino beams is fixed by their arrival zenith angle $\theta_{z}$ (with $\cos \theta_{z}=1$ for vertical

\footnotetext{
${ }^{2}$ For an expansion with solar mixing effects included, see Ref. [61].
} 

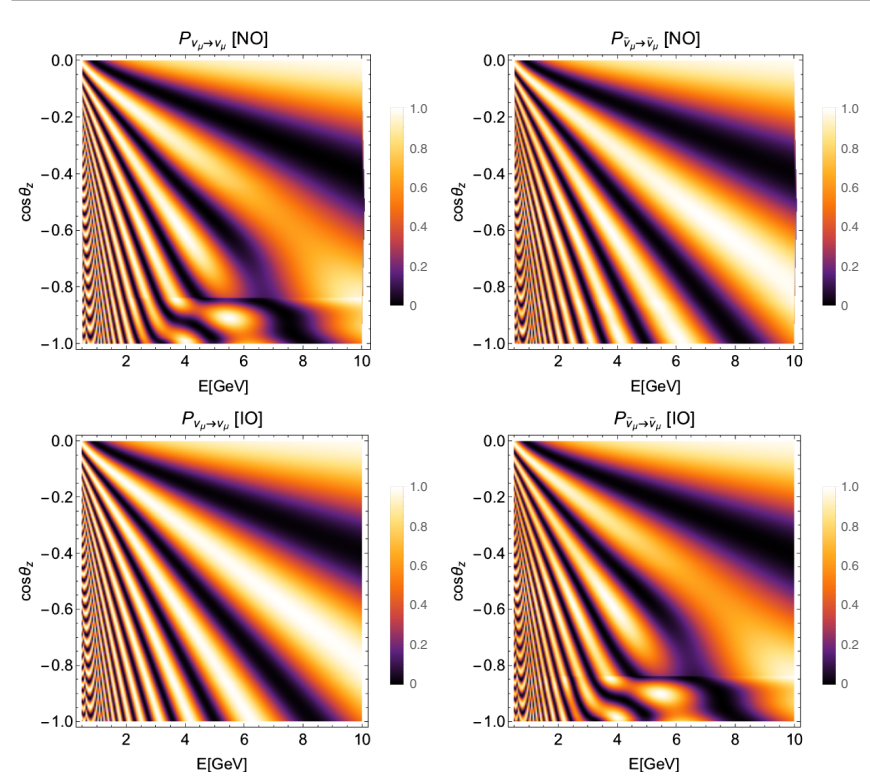

FIG. 1. Left panels: Survival probability $P\left(\nu_{\mu} \rightarrow \nu_{\mu}\right)$ as a function of the neutrino energy $E$ and the cosine of the zenith angle, $\cos \theta_{z}$, for normal (inverted) ordering in the top (bottom) line. Right panels: The same as in the left panels, but for the antineutrino channel.

downgoing neutrinos and $\cos \theta_{z}=-1$ for vertical upgoing neutrinos):

$L=R_{\oplus}\left(\sqrt{\left(1+\frac{h}{R_{\oplus}}\right)^{2}-\left(1-\cos \theta_{z}\right)^{2}}-\cos \theta_{z}\right)$,

with $R_{\oplus}$ Earth's radius and $h \simeq 15 \mathrm{~km}$ the neutrino production distance from Earth's surface. The dependence of the survival probabilities $P\left(\nu_{\mu} \rightarrow \nu_{\mu}\right)$ and $P\left(\bar{\nu}_{\mu} \rightarrow \bar{\nu}_{\mu}\right)$ on the neutrino energy $E$ and the cosine of the zenith angle, $\cos \theta_{z}$, is shown in the left and right panels in Fig. 1 for normal and inverted ordering (top and bottom figures), respectively. Notice that, in the case of normal ordering, the resonance takes place at the aforementioned energies (3-8 GeV) for almost vertical upgoing neutrinos, $-1<$ $\cos \theta_{z}<-0.8$, while for the inverted ordering, such a resonant enhancement in the transition probabilities will take place in the antineutrino channel instead. Therefore, even if both the angular and the energy resolution of the detector should be optimal, the key ingredient to disentangle matter effects (and, ultimately, the neutrino mass ordering) is to have a detector with muon charge tagging, generally achieved with a magnetized detector. However, as we shall shortly see, LArTPCs allow for such a possibility without the need of a magnetic field.

\section{ATMOSPHERIC NEUTRINO EVENTS IN DUNE: MUON CAPTURE IN ARGON}

Our statistical analyses will deal with three possible fully contained event samples at atmospheric neutrino detectors: $\mu^{-}$-like events that undergo muon capture $\left(N_{i, j, \mu}^{\mathrm{cap}}\right)$, the rest of the muons and all of the antimuons that undergo muon decay $\left(N_{i, j, \mu}^{\text {rest }}\right)$, and $e$-like events $\left(N_{i, j, e}\right) .^{3}$

Let us start with the $\mu^{-}$-like contained events produced by the interactions of atmospheric upgoing neutrinos in the LArTPC DUNE detector. In a LArTPC, both ionization charge [62,63] and scintillation light [64] information can be used to infer the neutrino and antineutrino content in a muon neutrino beam. This is possible by exploiting the signature of $\mu^{-}$capture on argon nuclei, only available for contained events. In argon, the effective $\mu^{-}$lifetime resulting from the competing decay and nuclear capture processes is given by

$$
\tau=\left(1 / \tau_{\text {cap }}+Q / \tau_{\text {free }}\right)^{-1},
$$

where $\tau_{\text {cap }}$ is the lifetime of the capture process, $Q=0.988$ is the Huff correction factor [65], and $\tau_{\text {free }}=2197.0 \mathrm{~ns}$ [66] is the muon lifetime in a vacuum. The resulting $\mu^{-}$ capture fraction is then given by

$$
\epsilon^{\text {cap }}=\tau / \tau_{\text {cap }}=1-\tau / \tau_{\text {free }} .
$$

The most precise determination of the $\mu^{-}$lifetime in argon was obtained in Ref. [67], $\tau=(616.9 \pm 6.7) \mathrm{ns,}$ resulting in

$$
\epsilon^{\mathrm{cap}}=(71.9 \pm 0.3) \%
$$

This measurement is fully compatible with the earlier measurement of $\tau=(606 \pm 29) \mathrm{ns}$ in Ref. [65] and the preliminary result from LArIAT of $\tau=(626 \pm 48)$ ns [68]. In our analysis, we use the central value and uncertainty in Eq. (8).

For our sensitivity estimates, we also assume a $100 \%$ efficiency for tagging Michel electrons and positrons from $\mu^{ \pm}$decays at rest, as done in Ref. [60]. Any tagging inefficiency would cause decay events to be misinterpreted as capture events and should, therefore, be avoided for optimal muon neutrino and antineutrino separation. We consider this approximation to be sufficient for the purposes of this feasibility study. Efficiency estimates using detailed DUNE simulations are not yet publicly available. Still, early data from ICARUS [69] and LArIAT [68] have already shown that the Michel electron tagging efficiency can reach values close to unity in LArTPC detectors using either charge or light information. In any case, any Michel electron tagging inefficiency smaller than $\left(1-\epsilon^{\text {cap }}\right) \simeq 28 \%$ will have a subdominant contribution to the mixing of muon neutrino and muon antineutrino stopping samples in

\footnotetext{
${ }^{3}$ Electron charge identification is impossible at $\mathrm{GeV}$ energies, and we shall consider just one event sample which accounts for both $e^{+}$and $e^{-}$-like events.
} 

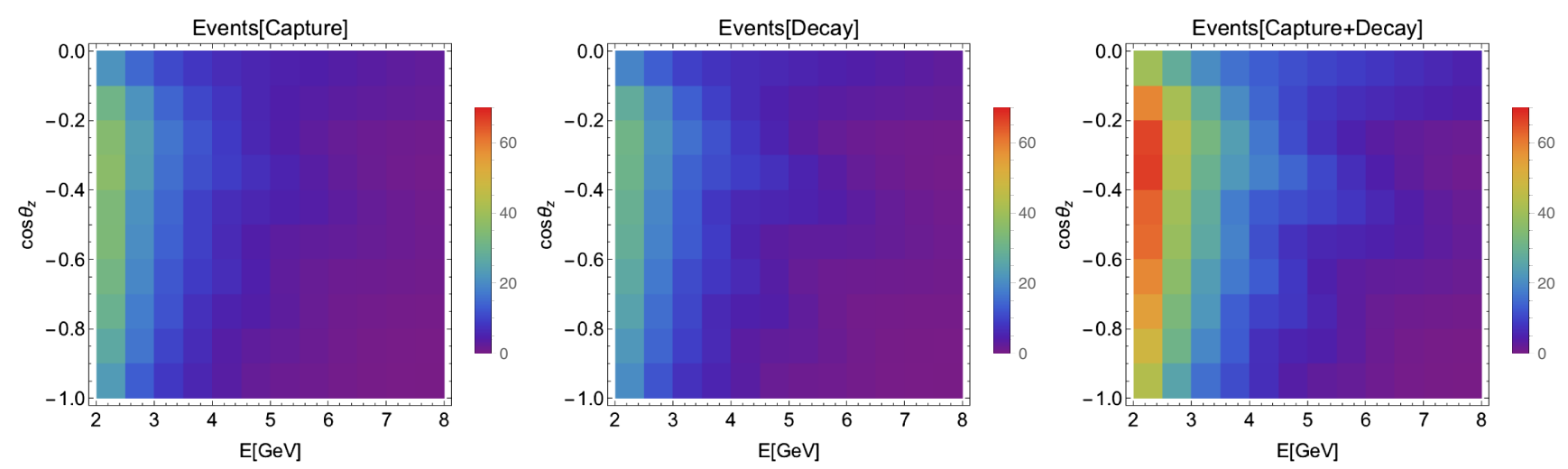

FIG. 2. Number of expected muon events after $400 \mathrm{kt} \cdot \mathrm{yr}$ exposure time, separating capture and decay events (first two panels) and combining all muon-type events (right panel), using the parameters in Table I and $\sin ^{2} \theta_{23}=0.547$.

our analysis, compared to the effect of $\mu^{-}$tracks that do not capture and decay.

Therefore, it appears possible to select a statistically significant, highly pure, sample of $\mu^{-}$-like atmospheric neutrino interactions, with an identification efficiency of $\epsilon^{\text {cap }}$, as given in Eq. (8). The number of muonlike contained events in the $i$ th neutrino energy $\left(E_{r}\right)$ and $j$ th cosine of the zenith angle $\left(c_{r, \nu}\right)$ bin (both reconstructed quantities) reads as

$$
\begin{aligned}
& N_{i, j, \mu^{-}\left(\mu^{+}\right)} \\
& =\frac{2 \pi N_{\mathrm{T}} t}{V_{\mathrm{det}}} \int_{E_{r, i}}^{E_{r, i+1}} d E_{r, \nu} \int_{c_{r, \nu, j}}^{c_{r, \nu, j+1}} d c_{r, \nu} \int_{0}^{\infty} d E_{\nu} \int_{-1}^{1} d c_{\nu} V_{\mu} \\
& \quad \times\left(\frac{d \phi_{\nu_{e}}\left(\nu_{\mu}\right)\left(\bar{\nu}_{e}\left(\bar{\nu}_{\mu}\right)\right)}{d E_{\nu} d \Omega} \sigma_{\nu_{\mu}\left(\bar{\nu}_{\mu}\right)}^{\mathrm{CC}} P_{\nu_{e}\left(\nu_{\mu}\right) \rightarrow \nu_{\mu}\left(\bar{\nu}_{e}\left(\bar{\nu}_{\mu}\right) \rightarrow \bar{\nu}_{\mu}\right)}\right) \\
& \quad \times R_{e}^{\mu}\left(E_{r, \nu}, E_{\nu}\right) R_{\theta}^{\mu}\left(\theta_{r, \nu}, \theta_{\nu}\right)
\end{aligned}
$$

where $d \phi_{\nu}$ 's are the atmospheric neutrino differential fluxes, $\sigma^{\mathrm{CC}}$ is the charged current $(\mathrm{CC})$ neutrino cross sections in argon, $N_{\mathrm{T}}$ is the number of available targets, $V_{\text {det }}$ is the total volume of the detector, $V_{\mu}$ is the effective detector volume, and $t$ is the exposure time. Finally, $R_{e}^{\mu}\left(E_{r, \nu}, E_{\nu}\right)$ and $R_{\theta}^{\mu}\left(\theta_{r, \nu}, \theta_{\nu}\right)$ account for the energy and angular smearing, respectively.

The $\mu^{-}$-like contained events that undergo muon capture are given by

$$
N_{i, j, \mu}^{\mathrm{cap}}=\epsilon^{\mathrm{cap}} N_{i, j, \mu^{-}},
$$

while the remaining muonlike events are given by

$$
N_{i, j, \mu}^{\mathrm{rest}}=\left(1-\epsilon^{\mathrm{cap}}\right) N_{i, j, \mu^{-}}+N_{i, j, \mu^{+}} .
$$

In Fig. 2, we show an example for the expected number of events, fixing the value the oscillation parameters to the ones in Table I and the atmospheric mixing angle to $\sin ^{2} \theta_{23}=0.547$. In the first two panels, we plot the capture and decay events separately, respectively, while showing the combination of both samples in the right panel.
Note that, when trying to reconstruct the right panel, there can be more degeneracies among parameters in the analysis than when fitting the two sets independently, and, therefore, one expects to obtain stronger results. This is indeed the case, as we will see below.

In the case of electrons, the number of $e$-like events in the $i$ th and $j$ th bins in $\left(E_{r}, c_{r, \nu}\right)$ reads as

$$
\begin{aligned}
& N_{i, j, e^{-}\left(e^{+}\right)} \\
& =2 \pi N_{\mathrm{T}} t \int_{E_{r, i}}^{E_{r, i+1}} d E_{r, \nu} \int_{c_{r, \nu, j}}^{c_{r, \nu, j+1}} d c_{r, \nu} \int_{0}^{\infty} d E_{\nu} \int_{-1}^{1} d c_{\nu} \\
& \quad \times\left(\frac{d \phi_{\nu_{e}\left(\nu_{\mu}\right)\left(\bar{\nu}_{e}\left(\bar{\nu}_{\mu}\right)\right)}}{d E_{\nu} d \Omega} \sigma_{\nu_{e}\left(\bar{\nu}_{e}\right)}^{\mathrm{CC}} P_{\nu_{e}\left(\nu_{\mu}\right) \rightarrow \nu_{e}\left(\bar{\nu}_{e}\left(\bar{\nu}_{\mu}\right) \rightarrow \bar{\nu}_{e}\right)}\right) \\
& \quad \times R_{e}^{e}\left(E_{r, \nu}, E_{\nu}\right) R_{\theta}^{e}\left(\theta_{r, \nu}, \theta_{\nu}\right) .
\end{aligned}
$$

As previously stated, we just consider one electronlike event sample $N_{i, j, e}$, which is computed as the sum of $N_{i, j, e^{-}}$ and $N_{i, j, e^{+}}$.

Regarding the atmospheric electron and muon (anti) neutrino fluxes, for the differential fluxes $\frac{d \phi_{\nu_{\alpha}}}{d E_{\nu} d \Omega}$ that appear in Eqs. (9) and (12), we use the results from Ref. [70], albeit very similar numbers would have been obtained using the fluxes from Refs. [71-73]. We shall comment in the following section on the errors on these atmospheric neutrino fluxes that have been properly added to other sources of systematic uncertainties in our numerical studies.

TABLE I. The oscillation parameters used to generate the mock data [1]. We use various values for the atmospheric angle $\theta_{23}$.

\begin{tabular}{lcc}
\hline \hline Parameter & Normal ordering & Inverted ordering \\
\hline$\Delta m_{21}^{2}$ & $7.55 \times 10^{-5} \mathrm{eV}^{2}$ & $7.55 \times 10^{-5} \mathrm{eV}^{2}$ \\
$\Delta m_{31}^{2}$ & $2.50 \times 10^{-3} \mathrm{eV}^{2}$ & $-2.42 \times 10^{-3} \mathrm{eV}^{2}$ \\
$\sin ^{2} \theta_{12}$ & 0.320 & 0.320 \\
$\sin ^{2} \theta_{13}$ & 0.02160 & 0.0222 \\
$\delta$ & 0 & 0 \\
\hline \hline
\end{tabular}


The cross sections for muon and electron (anti)neutrino interactions on argon nuclei in the $0-10 \mathrm{GeV}$ neutrino energy range have been simulated by means of the GENIE Monte Carlo neutrino event generator [74]. GENIE is extensively used by the neutrino physics community and by the DUNE Collaboration, in particular. As our cross section model, we use the total charged-current (anti)neutrino cross sections provided by GENIE version 2.12 .10 on ${ }^{40} \mathrm{Ar}$ nuclei (18 protons and 22 neutrons). The model accounts for a comprehensive list of interaction processes, including quasielastic scattering, baryon resonance production, coherent pion production in neutrino-nucleus scattering, and deep inelastic scattering. Nuclear effects affecting total cross sections are included. Final state hadronic interactions occurring within the argon target nucleus are not simulated but indirectly accounted for via our assumed energy and angular resolution functions.

To compute the effective volume fraction $V_{\mu} / V_{\text {det }}$ in Eq. (9) for contained muon events, we have approximated the DUNE detector to be made of four independent modules with approximately 13 kton of LAr active mass each, each of them assumed to have an elliptical cylindrical shape of $12 \mathrm{~m}$ height and major and minor axis of $a=$ $29 \mathrm{~m}$ and $b=7.25 \mathrm{~m}$, respectively. For the calculation of the effective volume, we have taken into account the muon range in argon, $R_{\mu}\left(E_{\mu}\right)$, which depends on the lepton energy. Conservatively, we have also computed the number of $\mu^{+}$-like events, restricting ourselves to the contained topology. This assumption eases the comparison with respect to the case in which no flavor tagging is available and ensures good energy reconstruction for the full muonlike event sample.

As for the energy and angular smearing inherent to reconstruction processes and final state hadronic interactions within $\operatorname{argon}$ nuclei, $R_{e}^{\mu}\left(E_{r, \nu}, E_{\nu}\right)$ and $R_{\theta}^{\mu}\left(\theta_{r, \nu}, \theta_{\nu}\right)$ in Eq. (9), and $R_{e}^{e}\left(E_{r, \nu}, E_{\nu}\right)$ and $R_{\theta}^{e}\left(\theta_{r, \nu}, \theta_{\nu}\right)$ in Eq. (12), are taken to be Gaussian functions. The assumed Gaussian widths $\sigma_{E} / E$ and $\sigma_{\theta}$ for $\nu_{e}, \bar{\nu}_{e}, \nu_{\mu}$, and $\bar{\nu}_{\mu}$ charged-current interactions on argon are shown in Fig. 3. We use the dashed curves in the figure to parametrize the resolutions as functions of the neutrino energy $E_{\nu}$, according to

$$
\begin{aligned}
\sigma_{E} / E_{\nu} & =A / E_{\nu}^{B}, \\
\sigma_{\theta} & =C / E_{\nu}+D .
\end{aligned}
$$

The numerical values for the parameters in Eq. (13) are reported in the Appendix. The resolution functions were obtained via fast Monte Carlo simulations as follows, similarly to what was done in Refs. [59,60]. First, large samples of monoenergetic neutrino-argon interactions are simulated with GENIE, for the various neutrino flavors $\left(\nu_{e}, \bar{\nu}_{e}, \nu_{\mu}\right.$, and $\left.\bar{\nu}_{\mu}\right)$ and for the relevant neutrino energy range $0.5-8 \mathrm{GeV}$. The GENIE simulation includes nuclear effects. Second, for each event, each final state particle exiting the nucleus has its kinetic energy and angular direction smeared according to the assumptions described
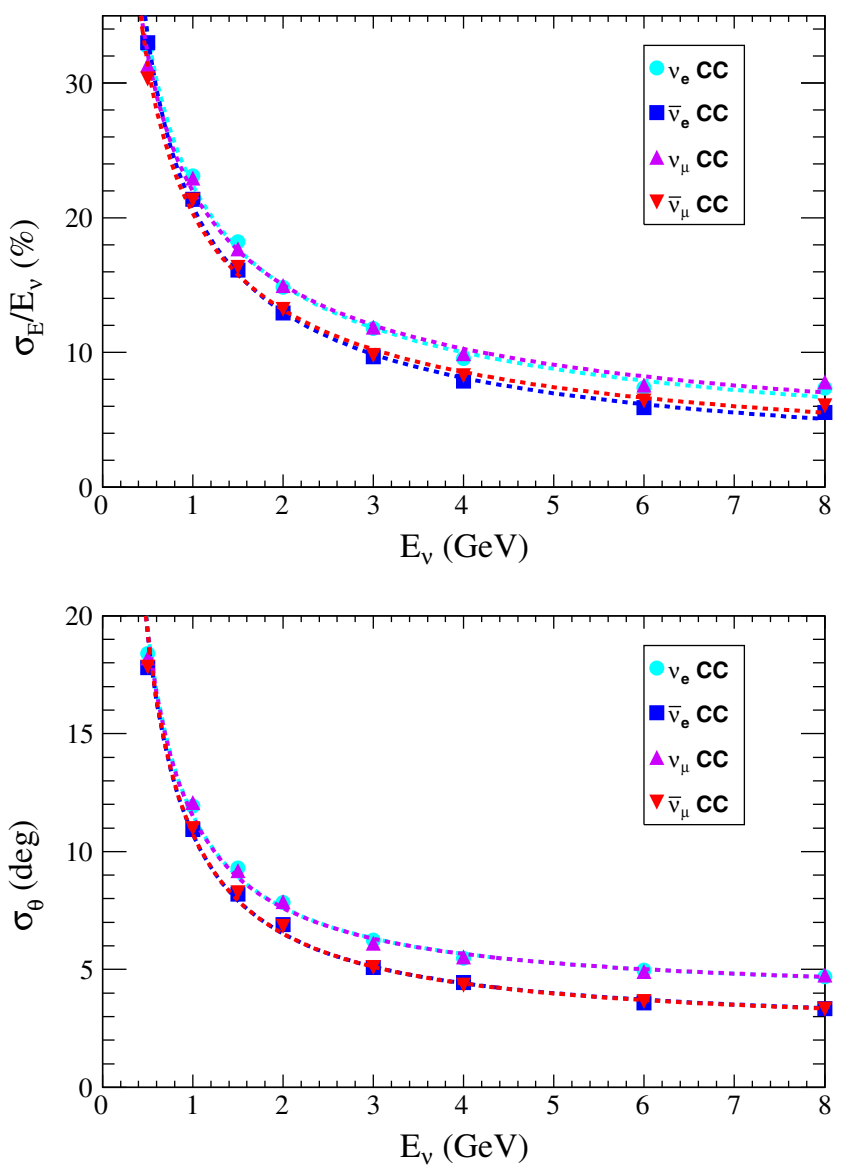

FIG. 3. Relative neutrino energy resolution $\sigma_{E} / E_{\nu}$ (top) and absolute neutrino angular resolution $\sigma_{\theta}$ (bottom) as a function of neutrino energy $E_{\nu}$ assumed in this study, for $\nu_{e}, \bar{\nu}_{e}, \nu_{\mu}$, and $\bar{\nu}_{\mu}$ charged-current interactions on argon nuclei.

in Ref. [60]. The relative energy resolutions are taken to be $1 \% / \sqrt{E_{e}}+1 \%$ and $3 \%$ and $30 \% / \sqrt{E_{\text {had }}}$ for electrons, muons, and hadrons, respectively, where $E_{e}$ and $E_{\text {had }}$ are expressed in $\mathrm{GeV}$. The absolute angular resolutions are taken to be $1^{\circ}, 1^{\circ}$, and $10^{\circ}$, respectively, for the same three final state particle categories and for all energies. Third, the incoming neutrino energy and direction of each interaction is reconstructed as follows:

$$
\begin{aligned}
E_{r, \nu} & =K_{r, l}+m_{l}+\sum_{h} K_{r, h}, \\
\theta_{r, \nu} & =\arccos \left(p_{z r, \nu} /\left|\vec{p}_{r, \nu}\right|\right),
\end{aligned}
$$

where $K_{r, l}$ and $K_{r, h}$ are the reconstructed charged lepton and hadron kinetic energies, respectively, $m_{l}$ is the charged lepton mass, the sum $\sum_{h}$ is intended over all final state hadrons, and $\vec{p}_{r, L} \equiv \vec{p}_{r, l}+\sum_{h} \vec{p}_{r, h}$ is the reconstructed 3 -momentum of the incoming neutrino, where the true neutrino direction is defined along the $z$ axis. Fourth, histograms of the reconstructed neutrino energy and direction are obtained for each (neutrino flavor, neutrino energy) simulated data sample. Fifth, $\sigma_{E} / E_{\nu}$ and $\sigma_{\theta}$ for each data 
sample are obtained from a Gaussian fit to the energy histogram and from the mean of the angle histogram, respectively. The resolution functions are shown in Fig. 3 for each sample via marker symbols. Sixth, the energy dependence of the resolutions functions is parametrized according to Eq. (13).

The behavior of the resolution functions in Fig. 3 can be easily understood. The main effect is that both $\sigma_{E} / E$ and $\sigma_{\theta}$ improve noticeably as the neutrino energy increases. For $\sigma_{E} / E$, this is a direct consequence of the relative energy resolutions assumed for electrons and (especially) hadrons, improving as the particle energies increase. For $\sigma_{\theta}$, this is due to the Fermi momentum of the target nucleon, whose angular smearing effect is more important at low neutrino energies. A second, smaller, effect can also be appreciated in Fig. 3, namely, that antineutrino resolutions are slightly better than neutrino ones, for both $\sigma_{E} / E$ and $\sigma_{\theta}$. On the one hand, this is due to the fact that the average inelasticity (or energy fraction carried away by final state hadrons) is somewhat lower in antineutrino interactions [75] and, on the other, because hadron resolutions are substantially worse than charged lepton ones. An even smaller difference can be appreciated between the relative energy resolutions of electron and muon antineutrinos of the same energy. In this case, electron antineutrino energy resolutions are slightly better because of the better assumed accuracy in reconstructing electron energy $\left(1 \% / \sqrt{E_{e}}+1 \%\right)$ compared to muon energy (3\%).

Our energy resolution assumptions in Fig. 3 are similar to the ones in Refs. $[59,60]$ that use similar methodologies and assumptions. They are qualitatively similar also to the ones obtained in more recent studies; see Refs. [75,76]. On the other hand, we are not aware of other neutrino angular resolutions studies in LArTPCs to compare our findings with.

\section{ANALYSES AND RESULTS}

Here, we describe the statistical analysis and how we extract the sensitivity to the neutrino mass ordering. In order to emphasize the impact of the muon capture in argon, we present two possible analyses. The first case will assume that no charge identification is possible. Then, we will focus on the extra bonus that the muon capture in argon process provides.

In the following, we define a fiducial mass ordering, true ordering $(T O)$, in order to generate mock data. Then, we try to reconstruct the event rates using the wrong ordering (WO) assumption. Although there is some preference for normal neutrino mass ordering, as previously stated, we shall also study the case of inverted ordering as TO.

We use Eqs. (9) and (12) to generate our mock data, using the oscillation parameters from Table I and assuming a $400 \mathrm{kt} \cdot \mathrm{yr}$ exposure. We will present our results as a function of the atmospheric angle $\theta_{23}$. Therefore, there is no fixed value for this angle in the table. Notice that, since our main sensitivity comes from the $\nu_{\mu} \rightarrow \nu_{\mu}$ channel, the effects of the $C P$-violating phase $\delta$ are negligible, and, therefore, we set $\delta=0$, finding very similar results for other values of the $C P$ phase.

Next, we try to reconstruct the event rates following the two methods mentioned above. Before presenting our results, let us discuss our treatment of systematic uncertainties. We consider several sources of systematic uncertainties in our analyses, coming from the fact that we do not have a perfect knowledge of the atmospheric flux and detector response. In particular, we include an overall rate normalization error accounting for both flux normalization and detector efficiency uncertainties, an error on the $\nu / \bar{\nu}$ atmospheric flux ratio, and an error on the $\nu_{\mu} / \nu_{e}$ atmospheric flux ratio. We follow Ref. [60] and assume a 15\%, $5 \%$, and $2 \%$ Gaussian error on these three quantities, respectively. We have verified that adding a systematic on the spectral index of the neutrino flux would have a negligible effect. As explained in the previous section, we also add an uncertainty on $\epsilon^{\text {cap}}$; see Eq. (8). Apart from the systematic uncertainties, we also marginalize over the oscillation parameters $\Delta m_{31}^{2}, \sin ^{2} \theta_{13}$, and $\sin ^{2} \theta_{23}$ within their current $3 \sigma$ ranges for both orderings, namely, $\left|\Delta m_{31}^{2}\right| \in[2.31,2.60] \times 10^{-3} \mathrm{eV}^{2}, \sin ^{2} \theta_{13} \in[0.0196,0.0244]$, and $\sin ^{2} \theta_{23} \in[0.455,0.599]$. It is well known that the solar parameters do not have big effects in atmospheric neutrino oscillations; hence, they are fixed to their best-fit values throughout the analysis.

\section{A. Method A: Analysis without muon capture tagging}

In this case, muons and antimuons cannot be distinguished. We therefore build a $\chi^{2}$ function in the following way:

$$
\chi_{A}^{2}\left(\sin ^{2} \theta_{23}^{\text {true }}\right)=\min _{\text {sys }, \Delta m_{31}^{2}, \theta_{13}, \theta_{23}}\left\{\chi_{\mu^{-}+\mu^{+}}^{2}+\chi_{e^{-}+e^{+}}^{2}\right\} .
$$

We use a Poissonian $\chi^{2}$, which for muons is

$\chi_{\mu^{-}+\mu^{+}}^{2}=2 \sum_{i, j} N_{i, j, \mu}^{\mathrm{WO}}-N_{i, j, \mu}^{\mathrm{TO}}+N_{i, j, \mu}^{\mathrm{TO}} \log \left(\frac{N_{i, j, \mu}^{\mathrm{TO}}}{N_{i, j, \mu}^{\mathrm{WO}}}\right)$,

where $N_{i, j, \mu}^{\mathrm{TO}(\mathrm{WO})}=N_{i, j, \mu^{+}}^{\mathrm{TO}(\mathrm{WO})}+N_{i, j, \mu^{-}}^{\mathrm{TO}(\mathrm{WO})}$ is the sum of the muon and antimuon contributions. The same formula applies to $\chi_{e^{-}+e^{+}}^{2}$, with the replacement $\mu \rightarrow e$. The results of our analysis with method A are shown as red curves in Fig. 4. Note that the sensitivity ranges between 1.5 and $3.5 \sigma$ approximately when normal ordering is the TO (solid lines) and between 1.5 and $2 \sigma$ for true inverted ordering (dashed line).

\section{B. Method B: Analysis with muon capture tagging}

In this other strategy, we use muon capture to distinguish $\sim 72 \%$ of the muons from antimuons. Therefore, this time our $\chi^{2}$ function contains three terms, namely, 


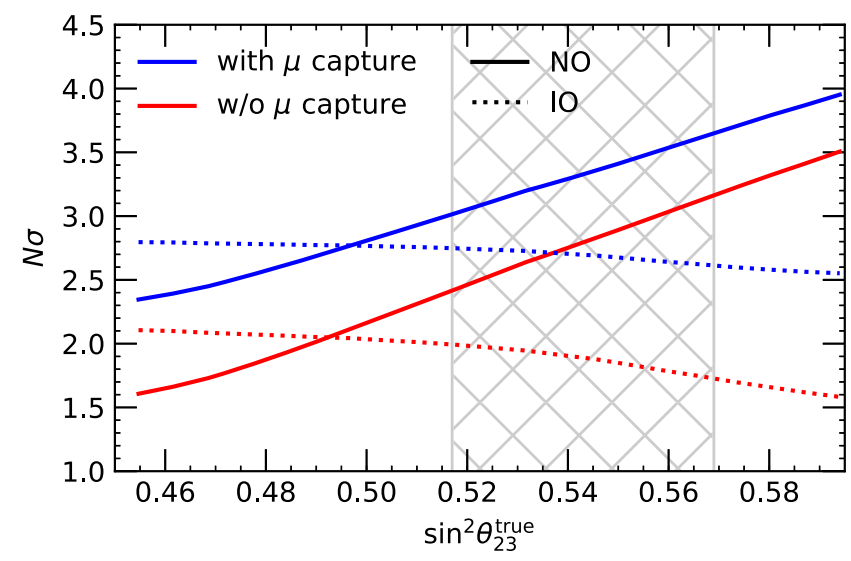

FIG. 4. The DUNE sensitivity to the neutrino mass ordering as a function of $\sin ^{2} \theta_{23}^{\text {true }}$. Red (blue) lines correspond to analysis method A (B). Solid lines are for normal ordering as true ordering, while dashed lines show the sensitivity in the case of true inverted ordering. The gray band corresponds to the current $1 \sigma$ region for the atmospheric angle.

$\chi_{B}^{2}\left(\sin ^{2} \theta_{23}^{\text {true }}\right)=\min _{\text {sys, } \Delta m_{31}^{2}, \theta_{13}, \theta_{23}}\left\{\chi_{\mu}^{2, \text { cap }}+\chi_{\mu}^{2, \text { rest }}+\chi_{e^{-}+e^{+}}^{2}\right\}$.

The electron term is the same as for method A, while the other two terms, corresponding to the events with muon capture (cap) and all other events (rest), are given by

$\chi_{\mu}^{2, X}=2 \sum_{i, j} N_{i, j, \mu}^{\mathrm{WO}, X}-N_{i, j, \mu}^{\mathrm{TO}, X}+N_{i, j, \mu}^{\mathrm{TO}, X} \log \left(\frac{N_{i, j, \mu}^{\mathrm{TO}, X}}{N_{i, j, \mu}^{\mathrm{WO}, X}}\right)$,

where $X \in\{$ cap, rest $\}$; see Eqs. (10) and (11). The results of the analysis with muon capture are shown in Fig. 4 by the blue curves. As before, true normal ordering is shown as a solid line, while the case of true inverted ordering is represented by a dashed line. The gray band in the figure represents the current $1 \sigma$ allowed region for $\sin ^{2} \theta_{23}$. Note how the sensitivity to the mass ordering is now at the $2.5-4 \sigma$ level, implying an important improvement with respect to the results obtained with method A. In particular, for the current best-fit point [1] we find that, using atmospheric neutrinos with muon capture, DUNE could measure the neutrino mass ordering at the $3.5 \sigma$ level. Our method B results can also be compared with the results in the DUNE conceptual design report [59], where a similar sensitivity reach and dependence on $\sin ^{2} \theta_{23}$ were obtained. Compared to Ref. [59], however, our results more clearly highlight the importance of the muon capture tag.

\section{CONCLUSIONS}

We have explored the advantages of muon capture on argon nuclei, a process that improves the sensitivity to the neutrino mass ordering using atmospheric neutrino events at the liquid argon time projection chamber DUNE far detector. This is a very relevant result, since it comes without any extra cost. Furthermore, it can be combined with DUNE beam neutrino results, allowing for an enhancement in the total sensitivity to the mass ordering determination. It is important to notice that our results are applicable to any experiment using argon. In the case of accelerator-based neutrinos, where significant $\nu_{\mu}$ contamination exists in the $\bar{\nu}_{\mu}$ beam, statistical neutrino and antineutrino separation based on muon capture could also be used to enhance DUNE oscillation sensitivities.

\section{ACKNOWLEDGMENTS}

This research made use of the MINUIT algorithm[77] via the iminuit [78] Python interface. We thank Peter Denton for useful comments on the draft. Work supported by the Spanish Grants No. FPA2015-68783-REDT, No. FPA2017-90566-REDC (Red Consolider MultiDark), No. FPA2017-85216-P, No. FPA2017-85985-P, No. FPA2017-82081-ERC, and No. SEV-2014-0398 (AEI/FEDER, UE, MINECO) and PROMETEO/2018/ 165 (Generalitat Valenciana). S. G. receives support from the European Union's Horizon 2020 research and innovation program under the Marie Skłodowska-Curie individual Grant Agreement No. 796941. O. M. and M. S. are also supported by the European Union's Horizon 2020 research and innovation program under the Marie SkłodowskaCurie Grant Agreements No. 690575 and No. 674896. C. A. T. is supported by the MINECO fellowship No. BES2015-073593. M. T. acknowledges financial support from MINECO through the Ramón y Cajal Contract No. RYC2013-12438.

\section{APPENDIX: NEUTRINO ENERGY AND ANGLE RESOLUTIONS}

Figure 3 shows our estimated neutrino energy $\sigma_{E} / E_{\nu}$ (top) and neutrino angle $\sigma_{\theta}$ (bottom) resolutions as a function of neutrino energy $E_{\nu}$, for charged-current neutrino

TABLE II. Numerical values for the parameters appearing in Eq. (13) and defining the energy dependence of the neutrino energy resolution assumed in this work.

\begin{tabular}{lcccc}
\hline \hline Parameter & $\nu_{e}$ & $\bar{\nu}_{e}$ & $\nu_{\mu}$ & $\bar{\nu}_{\mu}$ \\
\hline$A$ & 22.4 & 20.8 & 22.0 & 20.3 \\
$B$ & 0.582 & 0.680 & 0.548 & 0.625 \\
\hline \hline
\end{tabular}

TABLE III. Numerical values for the parameters appearing in Eq. (13) and defining the energy dependence of the neutrino angle resolution assumed in this work.

\begin{tabular}{lcccc}
\hline \hline Parameter & $\nu_{e}$ & $\bar{\nu}_{e}$ & $\nu_{\mu}$ & $\bar{\nu}_{\mu}$ \\
\hline$C$ & 7.85 & 8.42 & 7.79 & 8.46 \\
$D$ & 3.70 & 2.31 & 3.71 & 2.29 \\
\hline \hline
\end{tabular}


interactions on argon. The resolutions are parametrized according to Eq. (13). The $A$ and $B$ parameters describing the relative neutrino energy resolution (in percent) and the $C$ and $D$ parameters describing the neutrino angle resolution (in degrees) are given in Tables II and III, respectively. The parameters are given separately for each neutrino flavor: $\nu_{e}, \bar{\nu}_{e}, \nu_{\mu}$, and $\bar{\nu}_{\mu}$. The parameters in Eq. (13) assume that $E_{\nu}$ is expressed in $\mathrm{GeV}$.
[1] P. F. de Salas, D. V. Forero, C. A. Ternes, M. Tortola, and J. W. F. Valle, Status of neutrino oscillations 2018: $3 \sigma$ hint for normal mass ordering and improved $C P$ sensitivity, Phys. Lett. B 782, 633 (2018).

[2] S. Gariazzo, M. Archidiacono, P. F. de Salas, O. Mena, C. A. Ternes, and M. Tórtola, Neutrino masses and their ordering: Global data, priors and models, J. Cosmol. Astropart. Phys. 03 (2018) 011.

[3] P. F. De Salas, S. Gariazzo, O. Mena, C. A. Ternes, and M. Tórtola, Neutrino mass ordering from oscillations and beyond: 2018 status and future prospects, Front. Astron. Space Sci. 5, 36 (2018).

[4] L. Wolfenstein, Neutrino oscillations in matter, Phys. Rev. D 17, 2369 (1978).

[5] S. P. Mikheyev and A. Yu. Smirnov, Resonance amplification of oscillations in matter and spectroscopy of solar neutrinos, Sov. J. Nucl. Phys. 42, 913 (1985).

[6] S. J. Parke, Nonadiabatic Level Crossing in Resonant Neutrino Oscillations, Phys. Rev. Lett. 57, 1275 (1986).

[7] P. I. Krastev and A. Yu. Smirnov, Parametric effects in neutrino oscillations, Phys. Lett. B 226, 341 (1989).

[8] E. Akhmedov, P. Lipari, and M. Lusignoli, Matter effects in atmospheric neutrino oscillations, Phys. Lett. B 300, 128 (1993).

[9] S. T. Petcov, Diffractive-like (or parametric resonance-like?) enhancement of the Earth (day-night) effect for solar neutrinos crossing the Earth core, Phys. Lett. B 434, 321 (1998).

[10] M. Chizhov, M. Maris, and S. T. Petcov, On the oscillation length resonance in the transitions of solar and atmospheric neutrinos crossing the Earth core, arXiv:hep-ph/9810501.

[11] E. K. Akhmedov, Parametric resonance of neutrino oscillations and passage of solar and atmospheric neutrinos through the Earth, Nucl. Phys. B538, 25 (1999).

[12] M. V. Chizhov and S. T. Petcov, New Conditions for a Total Neutrino Conversion in a Medium, Phys. Rev. Lett. 83, 1096 (1999).

[13] M. V. Chizhov and S. T. Petcov, Enhancing mechanisms of neutrino transitions in a medium of nonperiodic constant density layers and in the Earth, Phys. Rev. D 63, 073003 (2001).

[14] B. Abi et al. (DUNE Collaboration), The DUNE far detector interim design report volume 1: Physics, technology and strategies, arXiv:1807.10334.

[15] B. Abi et al. (DUNE Collaboration), The DUNE far detector interim design report, volume 2: Single-phase module, arXiv: 1807.10327.
[16] B. Abi et al. (DUNE Collaboration), The DUNE far detector interim design report, volume 3: Dual-phase module, arXiv: 1807.10340.

[17] K. Abe et al. (T2K Collaboration), Search for $C P$ Violation in Neutrino and Antineutrino Oscillations by the T2K Experiment with $2.2 \times 10^{21}$ Protons on Target, Phys. Rev. Lett. 121, 171802 (2018).

[18] M. Friend (T2K Collaboration), Updated results from the T2K experiment with $3.13 \times 10^{2} 1$ protons on target, https:// t2k.org/docs/talk/335 (2019).

[19] M. A. Acero et al. (NOvA Collaboration), New constraints on oscillation parameters from $\nu_{e}$ appearance and $\nu_{\mu}$ disappearance in the NOvA experiment, Phys. Rev. D 98, 032012 (2018).

[20] A. Sousa (NOvA Collaboration), Implementation of Feldman-Cousins corrections and oscillation calculations in the HPC environment for the NOvA experiment, http:// nova-docdb.fnal.gov/cgi-bin/ShowDocument?docid=30696 (2018).

[21] S. Razzaque and A. Yu. Smirnov, Super-PINGU for measurement of the leptonic $C P$-phase with atmospheric neutrinos, J. High Energy Phys. 05 (2015) 139.

[22] K. J. Kelly, P. A. Machado, I. Martinez Soler, S. J. Parke, and Y. F. Perez Gonzalez, Sub-GeV Atmospheric Neutrinos and $C P$-Violation in DUNE, Phys. Rev. Lett. 123, 081801 (2019).

[23] M. C. Banuls, G. Barenboim, and J. Bernabeu, Medium effects for terrestrial and atmospheric neutrino oscillations, Phys. Lett. B 513, 391 (2001).

[24] J. Bernabeu, S. Palomares Ruiz, and S. T. Petcov, Atmospheric neutrino oscillations, $\theta_{13}$ and neutrino mass hierarchy, Nucl. Phys. B669, 255 (2003).

[25] T. Tabarelli de Fatis, Prospects of measuring $\sin ^{2} 2 \Theta_{13}$ and the sign of $\Delta m^{2}$ with a massive magnetized detector for atmospheric neutrinos, Eur. Phys. J. C 24, 43 (2002).

[26] A. B. Sousa (MINOSMINOS+Collaborations), First MINOS + data and new results from MINOS, AIP Conf. Proc. 1666, 110004 (2015).

[27] http://www.ino.tifr.res.in/ino/.

[28] S. Palomares-Ruiz and S. T. Petcov, Three-neutrino oscillations of atmospheric neutrinos, $\theta_{13}$, neutrino mass hierarchy and iron magnetized detectors, Nucl. Phys. B712, 392 (2005).

[29] D. Indumathi and M. V. N. Murthy, Question of hierarchy: Matter effects with atmospheric neutrinos and antineutrinos, Phys. Rev. D 71, 013001 (2005).

[30] R. Gandhi, P. Ghoshal, S. Goswami, P. Mehta, and S. Uma Sankar, Probing the nu mass hierarchy via atmospheric 
$\nu_{\mu}+\bar{\nu}_{\mu}$ survival rates in megaton water Cerenkov detectors, arXiv:hep-ph/0506145.

[31] S. T. Petcov and T. Schwetz, Determining the neutrino mass hierarchy with atmospheric neutrinos, Nucl. Phys. B740, 1 (2006).

[32] P. Huber, M. Maltoni, and T. Schwetz, Resolving parameter degeneracies in long-baseline experiments by atmospheric neutrino data, Phys. Rev. D 71, 053006 (2005).

[33] A. Samanta, The Mass hierarchy with atmospheric neutrinos at INO, Phys. Lett. B 673, 37 (2009).

[34] R. Gandhi, P. Ghoshal, S. Goswami, P. Mehta, S. U. Sankar, and S. Shalgar, Mass hierarchy determination via future atmospheric neutrino detectors, Phys. Rev. D 76, 073012 (2007).

[35] R. Gandhi, P. Ghoshal, S. Goswami, and S. U. Sankar, Resolving the mass hierarchy with atmospheric neutrinos using a liquid argon detector, Phys. Rev. D 78, 073001 (2008).

[36] A. Samanta, Discrimination of mass hierarchy with atmospheric neutrinos at a magnetized muon detector, Phys. Rev. D 81, 037302 (2010).

[37] V. Barger, R. Gandhi, P. Ghoshal, S. Goswami, D. Marfatia, S. Prakash, S. K. Raut, and S. U. Sankar, Neutrino Mass Hierarchy and Octant Determination with Atmospheric Neutrinos, Phys. Rev. Lett. 109, 091801 (2012).

[38] A. Ghosh, T. Thakore, and S. Choubey, Determining the neutrino mass hierarchy with INO, T2K, NOvA and reactor experiments, J. High Energy Phys. 04 (2013) 009.

[39] M. Blennow and T. Schwetz, Identifying the neutrino mass ordering with INO and NOvA, J. High Energy Phys. 08 (2012) 058; 11 (2012) 98.

[40] A. Ghosh and S. Choubey, Measuring the mass hierarchy with muon and hadron events in atmospheric neutrino experiments, J. High Energy Phys. 10 (2013) 174.

[41] M. M. Devi, T. Thakore, S. K. Agarwalla, and A. Dighe, Enhancing sensitivity to neutrino parameters at INO combining muon and hadron information, J. High Energy Phys. 10 (2014) 189.

[42] K. Abe et al. (Super-Kamiokande Collaboration), Atmospheric neutrino oscillation analysis with external constraints in Super-Kamiokande I-IV, Phys. Rev. D 97, 072001 (2018).

[43] O. Mena, I. Mocioiu, and S. Razzaque, Neutrino mass hierarchy extraction using atmospheric neutrinos in ice, Phys. Rev. D 78, 093003 (2008).

[44] M. G. Aartsen et al. (IceCube Collaboration), Probing the neutrino mass ordering with atmospheric neutrinos from three years of IceCube DeepCore data, arXiv:1902.07771.

[45] S. Adrian-Martinez et al. (KM3Net Collaboration), Letter of intent for KM3NeT 2.0, J. Phys. G 43, 084001 (2016).

[46] F. Capozzi, E. Lisi, and A. Marrone, Probing the neutrino mass ordering with KM3NeT-ORCA: Analysis and perspectives, J. Phys. G 45, 024003 (2018).

[47] S.-F. Ge, K. Hagiwara, and C. Rott, A novel approach to study atmospheric neutrino oscillation, J. High Energy Phys. 06 (2014) 150.

[48] S.-F. Ge and K. Hagiwara, Physics reach of atmospheric neutrino measurements at PINGU, J. High Energy Phys. 09 (2014) 024.
[49] M. G. Aartsen et al. (IceCube-PINGU Collaboration), Letter of intent: The precision IceCube next generation upgrade (PINGU), arXiv:1401.2046.

[50] F. Capozzi, E. Lisi, and A. Marrone, PINGU and the neutrino mass hierarchy: Statistical and systematic aspects, Phys. Rev. D 91, 073011 (2015).

[51] E. K. Akhmedov, S. Razzaque, and A. Yu. Smirnov, Mass hierarchy, 2-3 mixing and $C P$-phase with Huge Atmospheric Neutrino Detectors, J. High Energy Phys. 02 (2013) 082; 07 (2013) 26.

[52] S. K. Agarwalla, T. Li, O. Mena, and S. Palomares-Ruiz, Exploring the Earth matter effect with atmospheric neutrinos in ice, arXiv:1212.2238.

[53] M. Ribordy and A. Y. Smirnov, Improving the neutrino mass hierarchy identification with inelasticity measurement in PINGU and ORCA, Phys. Rev. D 87, 113007 (2013).

[54] D. Franco, C. Jollet, A. Kouchner, V. Kulikovskiy, A. Meregaglia, S. Perasso, T. Pradier, A. Tonazzo, and V. Van Elewyck, Mass hierarchy discrimination with atmospheric neutrinos in large volume ice/water Cherenkov detectors, J. High Energy Phys. 04 (2013) 008.

[55] W. Winter, Neutrino mass hierarchy determination with IceCube-PINGU, Phys. Rev. D 88, 013013 (2013).

[56] M. Blennow, P. Coloma, P. Huber, and T. Schwetz, Quantifying the sensitivity of oscillation experiments to the neutrino mass ordering, J. High Energy Phys. 03 (2014) 028.

[57] M. Blennow and T. Schwetz, Determination of the neutrino mass ordering by combining PINGU and Daya Bay II, J. High Energy Phys. 09 (2013) 089.

[58] M. G. Aartsen et al. (IceCube-PINGU Collaboration), PINGU sensitivity to the neutrino mass hierarchy, in Proceedings of the Cosmic Frontier Workshop: Snowmass 2013, Menlo Park, USA (2013).

[59] R. Acciarri et al. (DUNE Collaboration), Long-baseline neutrino facility (LBNF) and deep underground neutrino experiment (DUNE), arXiv:1512.06148.

[60] C. Adams et al. (LBNE Collaboration), The long-baseline neutrino experiment: Exploring fundamental symmetries of the universe, in Proceedings of Snowmass 2013: Workshop on Energy Frontier Seattle, USA (2013).

[61] E. K. Akhmedov, R. Johansson, M. Lindner, T. Ohlsson, and T. Schwetz, Series expansions for three flavor neutrino oscillation probabilities in matter, J. High Energy Phys. 04 (2004) 078.

[62] F. Cavanna, M. Kordosky, J. Raaf, and B. Rebel (LArIAT Collaboration), LArIAT: Liquid argon in a Testbeam, arXiv: 1406.5560.

[63] R. Acciarri et al. (MicroBooNE Collaboration), Michel electron reconstruction using cosmic-ray data from the MicroBooNE LArTPC, J. Instrum. 12, P09014 (2017).

[64] M. Sorel, Expected performance of an ideal liquid argon neutrino detector with enhanced sensitivity to scintillation light, J. Instrum. 9, P10002 (2014).

[65] T. Suzuki, D. F. Measday, and J. P. Roalsvig, Total nuclear capture rates for negative muons, Phys. Rev. C 35, 2212 (1987).

[66] M. Tanabashi et al. (Particle Data Group), Review of particle physics, Phys. Rev. D 98, 030001 (2018). 
[67] A. V. Klinskikh, S. Brianson, V. B. Brudanin, V. G. Egorov, C. Petitjean, and M. V. Shirchenko, Muon capture in Ar. The muon lifetime and yields of $\mathrm{Cl}$ isotopes, Bull. Russ. Acad. Sci.: Phys. 72, 735 (2008).

[68] W. M. Foreman, A demonstration of light-augmented calorimetry for low-energy electrons in liquid argon, $\mathrm{Ph}$. D. thesis, Chicago University, 2019.

[69] S. Amoruso et al. (ICARUS Collaboration), Measurement of the $\mu$ decay spectrum with the ICARUS liquid argon TPC, Eur. Phys. J. C 33, 233 (2004).

[70] G. D. Barr, T. K. Gaisser, P. Lipari, S. Robbins, and T. Stanev, Three-dimensional calculation of atmospheric neutrinos, Phys. Rev. D 70, 023006 (2004).

[71] M. Honda, T. Kajita, K. Kasahara, S. Midorikawa, and T. Sanuki, Calculation of atmospheric neutrino flux using the interaction model calibrated with atmospheric muon data, Phys. Rev. D 75, 043006 (2007).

[72] M. Honda, T. Kajita, K. Kasahara, and S. Midorikawa, Improvement of low energy atmospheric neutrino flux calculation using the JAM nuclear interaction model, Phys. Rev. D 83, 123001 (2011).

[73] M. Honda, M. Sajjad Athar, T. Kajita, K. Kasahara, and S. Midorikawa, Atmospheric neutrino flux calculation using the NRLMSISE-00 atmospheric model, Phys. Rev. D 92, 023004 (2015).

[74] C. Andreopoulos et al., The GENIE neutrino Monte Carlo generator, Nucl. Instrum. Methods Phys. Res., Sect. A 614, 87 (2010).

[75] A. Friedland and S. W. Li, Understanding the energy resolution of liquid argon neutrino detectors, Phys. Rev. D 99, 036009 (2019).

[76] V. De Romeri, E. Fernandez-Martinez, and M. Sorel, Neutrino oscillations at DUNE with improved energy reconstruction, J. High Energy Phys. 09 (2016) 030.

[77] F. James and M. Roos, Minuit-A system for function minimization and analysis of the parameter errors and correlations, Comput. Phys. Commun. 10, 343 (1975).

[78] iminuit team, iminuit-A python interface to minuit, https:// github.com/iminuit/iminuit. Accessed: 2018-03-05. 